\title{
Estudos de prevalência da brucelose bovina no âmbito do Programa Nacional de Controle e Erradicação de Brucelose e Tuberculose: Introdução
}

\author{
[Prevalence studies on bovine brucellosis according to Brazilian National Program for the \\ Control and Eradication of Bovine Brucellosis and Tuberculosis: Introduction] \\ F. Poester ${ }^{1}$, V.C.F. Figueiredo ${ }^{2}$, J.R. Lôbo ${ }^{2}$, V.S.P. Gonçalves ${ }^{1,3}$, A.P. Lage $^{1,4}$, E. Roxo ${ }^{1,5}$, \\ P.M.P.C. Mota ${ }^{1,6}$, E.E. Müller ${ }^{1,7}$, J.S. Ferreira Neto ${ }^{1,8^{*}}$ \\ ${ }^{1}$ Comitê Científico Consultivo para Brucelose e Tuberculose - MAPA - Brasília, DF \\ ${ }^{2}$ Departamento de Saúde Animal - MAPA - Brasília, DF \\ ${ }^{3}$ Faculdade de Agronomia e Medicina Veterinária - UnB - Brasília, DF \\ ${ }^{4}$ Escola de Veterinária - UFMG - Belo Horizonte, MG \\ ${ }^{5}$ Instituto Biológico de São Paulo - São Paulo, SP \\ ${ }^{6}$ Laboratório Nacional Agropecuário - MAPA - Pedro Leopoldo, MG \\ ${ }^{7}$ Faculdade de Medicina Veterinária - UEL - Londrina, PR \\ ${ }^{8}$ Faculdade de Medicina Veterinária e Zootecnia - USP - São Paulo, SP
}

\begin{abstract}
RESUMO
As estratégias de combate à brucelose bovina são bastante conhecidas e, até o momento, os resultados são divergentes. No Brasil, o Ministério da Agricultura, Pecuária e Abastecimento (MAPA) implementou, em 2001, o Programa Nacional de Controle e Erradicação da Brucelose e Tuberculose. Conhecer a situação epidemiológica da brucelose no início de um programa de controle permite: 1 escolher as melhores estratégias de controle em função da frequência e padrão de distribuição da doença na população e 2 - acompanhar o programa com a finalidade de promover correções e evitar o desperdício de tempo e de recursos. Em razão disso, é necessário realizar estudos para dar suporte à escolha das melhores estratégias para os vários estados e regiões brasileiras e criar um mecanismo racional de verificação da efetividade das ações implementadas. Para tanto, o MAPA estabeleceu um Termo de Cooperação Técnica com a Faculdade de Medicina Veterinária da Universidade de São Paulo e envolveu também a Faculdade de Agronomia e Veterinária da Universidade de Brasília. Até o momento, foram concluídos os estudos de 15 unidades federativas, cujos resultados são apresentados nos artigos subsequentes. Além disso, há um $16^{\circ}$ artigo que explora o impacto da vacinação de bezerras com a B19 na redução da prevalência da brucelose.
\end{abstract}

Palavras-chave: brucelose bovina, programa de erradicação, Brasil

\begin{abstract}
Although strategies for controlling and eradicating of bovine brucellosis are well known, the achievements vary widely. In 2001, the Brazilian Ministry of Agriculture, Livestock, and Food Supply (MAPA) started a new National Program for the Control and Eradication of Brucellosis and Tuberculosis (NPCEBT). In the beginning of a brucellosis control program, it is of utmost importance to gain insights into the epidemiological status of the disease in order to: (1) determine the sanitary measures according to the frequency and distribution patterns of the disease in the population; (2) monitor the development of the program and decide when to make adjustments, avoiding the waist of time and resources. As the epidemiological status of bovine brucellosis is not adequately known in Brazil, there was a case for undertaking cross-sectional studies targeted at identifying the best strategies
\end{abstract}

Recebido em 27 de março de 2009

Aceito em 23 de setembro de 2009

* Autor para correspondência (corresponding author)

E-mail: jsoares@vps.fmvz.usp.br 
for each state and region, as well as providing a basis for future appraisal of the NPCEBT. For this purpose, MAPA established a collaborative project with FMVZ-Universidade de São Paulo, which also included FAV-Universidade de Brasília. So far, 15 states completed the survey, whose results are described in the following papers. In addition, it is presented a paper that deals with modeling the impact of vaccination of heifers with strain 19 upon the reduction of brucellosis prevalence.

\section{Keywords: bovine brucellosis, national eradication program, Brazil}

A brucelose é uma antropozoonose conhecida desde épocas remotas. Há registros de que Hipócrates, em 460 A.C., fazia referência a pacientes com sintomas compatíveis com brucelose. Pesquisas recentes, realizadas na Itália, revelaram que esqueletos remanescentes de pessoas que sucumbiram à catástrofe do vulcão Vesúvio, na cidade de Herculano, ocorrida no ano 79 da era cristã, apresentavam lesões ósseas típicas de brucelose. Uma provável explicação para esse fato foi proporcionada pela microscopia eletrônica que revelou a presença de cocobacilos compatíveis com Brucella em queijos elaborados com leite de cabras e que foram encontrados carbonizados em escavações naquela cidade italiana (Capasso, 2002).

Em 1887, Sir David Bruce isolou o agente Micrococcus melitensis -, responsável pela febre de malta de soldados britânicos mortos em consequência dessa enfermidade na Ilha de Malta, localizada no Mediterrâneo, não muito distante da extinta cidade de Herculano (Godfroid et al., 2005). Em 1895, o patologista veterinário dinamarquês L. F. Bernhard Bang isolou uma bactéria de fetos abortados de bovinos, denominada Bacillus abortus, e identificou $o$ agente etiológico do aborto enzoótico dos bovinos (Nicoletti, 2002). Esses microrganismos foram posteriormente renomeados como Brucella melitensis, em homenagem ao seu descobridor, e Brucella abortus (León, 1994).

O gênero Brucella é composto por nove espécies (Foster et al., 2007; Scholz et al., 2008). B. melitensis, B. suis e B. abortus são espécies lisas, altamente patogênicas e responsáveis por doenças graves, principalmente em caprinos e ovinos, suínos e bovinos, respectivamente, assim como no homem (Corbel et al., 2006). As duas únicas espécies naturalmente rugosas são $B$. canis, causadora da brucelose canina $\mathrm{e}$ considerada a menos patogênica para o homem, e B. ovis, que só foi encontrada infectando naturalmente ovinos (Corbel, 1997). As espécies
B. neotomae e B. microti, isoladas de roedores silvestres, não são consideradas zoonóticas (Corbel, 1997; Scholz et al., 2008). B. ceti e B. pinnipedialis, patogênicas para mamíferos marinhos (Foster et al., 2007), já foram associadas a granulomas intracerebrais em pacientes com neurobrucelose (Sohn et al., 2003), osteomielite da coluna vertebral (McDonald et al., 2006) e a acidentes laboratoriais (Brew et al., 1999).

As infecções brucélicas nos animais domésticos estão associadas principalmente a problemas reprodutivos como abortamentos, nascimento de crias fracas e baixa fertilidade, com efeitos desastrosos para a pecuária. Assim, por causar prejuízo à pecuária e por ser transmitida dos animais para o homem, desde o início do século $\mathrm{XX}$, muitos países têm adotado medidas severas de controle ou erradicação da brucelose na população animal.

Para a brucelose bovina, as estratégias de combate são bastante conhecidas e podem ser resumidas em vacinação, certificação de propriedades livres por rotinas de testes indiretos, controle da movimentação de animais e sistema de vigilância específico. Os resultados alcançados pelos países, segundo os programas de controle, variam muito, pois há registros de sucessos e fracassos citados na literatura especializada.

Os programas bem estruturados e administrados atingem bons índices de controle, com redução significativa da prevalência, depois de aproximadamente 20 anos de trabalho. São programas laboriosos, que demandam ações bem coordenadas dos serviços oficiais e privados, e que trazem, como resultado, não só a eliminação da brucelose bovina, mas também a organização, fortalecimento e amadurecimento dos serviços de saúde animal, bem como a modernização da cadeia produtiva de carne e leite. 
Dinamarca, Finlândia, Suécia, Noruega, Áustria, Alemanha, Holanda e Luxemburgo, países situados ao norte do continente europeu, já receberam a qualificação de livres de brucelose bovina. França, Grécia, Irlanda, Itália, Portugal e Espanha, embora ainda não tenham sido declarados livres de brucelose bovina, encontram-se em fase adiantada de erradicação (Godfroid e Käsbohrer, 2002).

$\mathrm{Na}$ Grã-Bretanha, a brucelose bovina foi erradicada em 1979, e o país foi reconhecido como livre da doença desde 1985 . Nesse país, a doença foi reintroduzida em várias ocasiões, especialmente por meio de gado importado, sendo, porém detectada pelo sistema de vigilância, que inclui testes mensais em amostras de leite nos rebanhos leiteiros, testes sorológicos periódicos em gado de corte, controle rigoroso da parição em animais importados e investigação minuciosa de abortos (McGiven et al., 2008).

Os Estados Unidos da América iniciaram o combate à brucelose em 1934, como parte do programa de redução da população bovina, necessária em razão da grande depressão econômica pela qual o país atravessava na época. Até então, a prevalência da enfermidade entre os bovinos era de $11,5 \%$. O programa de controle foi organizado e posto em prática pelo governo federal, pelos governos estaduais e pelos produtores de carne e leite. Como resultado, em dezembro de 2000 não havia mais registros de rebanhos afetados por brucelose no país (Ragan, 2002).

Nos países em desenvolvimento, a situação não é tão favorável. O México, endêmico para a brucelose, começou a combatê-la em 1942, mas, apesar de alguns avanços obtidos ao longo dos anos, a situação ainda está longe de ser a ideal. Além do mais, nesse país existe a presença da $B$. melitensis, mais patogênica para o homem (Luna-Martinez e Mejía-Teran, 2002). Na América Central, a prevalência da brucelose bovina tem sido estimada entre 4 e $8 \%$ e programas baseados em vacinação e remoção de reagentes pouco têm contribuído para o avanço no controle da enfermidade (Moreno, 2002). No Paraguai, testes sorológicos realizados em 1,2 milhões de amostras, no período de 20 anos (1979-2000), indicaram que a quantidade de animais reagentes permaneceu constante entre 3 e 4\% (Baumgarten, 2002). Na Argentina, diversos estudos têm demonstrado que a brucelose está presente na maioria das espécies domésticas. Estima-se que a prevalência da brucelose bovina em propriedades seja de 10 a $13 \%$ e que 4 a $5 \%$ dos animais estejam infectados, o que resulta em perda anual calculada ao redor de 60 milhões de dólares (Samartino, 2002).

No Brasil, o Ministério da Agricultura, Pecuária e Abastecimento (MAPA), ao verificar a ineficácia das medidas até então adotadas, elaborou e lançou, no início de 2001, o Programa Nacional de Controle e Erradicação da Brucelose e Tuberculose (PNCEBT). Trata-se de um programa harmonizado com as condutas preconizadas pelos organismos internacionais e suficientemente flexível a ponto de permitir a sua implementação nos heterogêneos estados brasileiros (Brasil, 2006).

Quando se pretende iniciar um programa de controle de brucelose bovina, é de extrema importância conhecer a situação epidemiológica da doença, por duas razões principais: (1) permitir a escolha das melhores estratégias tendo em vista a frequência e distribuição da doença nas populações estudadas; (2) permitir que seja feito o acompanhamento do programa com vistas a possíveis correções, para evitar desperdício de tempo e de recursos.

No Brasil, são raros os estudos bem planejados e de grande abrangência sobre a situação da brucelose bovina. O último estudo nacional, que envolveu 19 estados, foi realizado em 1975 (Brasil, 1977). Depois disso, apenas cinco estados realizaram trabalhos que envolveram todo o seu território (Poester et al., 2002; Paulin e Ferreira Neto, 2003), com uso de diferentes metodologias. Portanto, a situação epidemiológica da brucelose bovina no Brasil não é adequadamente conhecida.

Assim, considerando a importância do PNCEBT para a cadeia produtiva de carne e leite, para a segurança dos consumidores de produtos de origem animal, para a imagem que o País projeta no mercado mundial e tendo em vista os altos custos inerentes aos procedimentos necessários para se atingirem os objetivos do programa, julgou-se necessária a realização de estudos que visassem elucidar a situação epidemiológica 
dessa zoonose no rebanho bovino brasileiro, indicar as melhores condutas e estratégias para os vários estados e regiões e criar um mecanismo de verificação da efetividade das ações implementadas.

Para tanto, foi estabelecido um termo de cooperação técnica entre o MAPA e a Faculdade de Medicina Veterinária e Zootecnia da Universidade de São Paulo, publicado no Diário Oficial da União em 1 de janeiro de 2003, cujo objetivo foi desenvolver estudos epidemiológicos no âmbito do PNCEBT, sendo um deles a caracterização da situação epidemiológica da brucelose bovina nas unidades federativas (UF) brasileiras. A Faculdade de Agronomia e Medicina Veterinária da Universidade de Brasília também integrou o grupo, e é necessário destacar que, em vários estados, houve a colaboração importante de laboratórios estaduais e de pesquisadores de outras universidades, nas quais estes estudos deram origem a projetos bem sucedidos de pósgraduação.

A adesão das UF ao estudo de caracterização epidemiológica da brucelose bovina foi espontânea e o aporte de recursos federais ocorreu sempre que necessário. O ritmo do trabalho de campo foi sempre ditado pela capacidade operacional de cada UF e em todos os casos desenvolveu-se com rapidez e eficiência. Hoje, já foram concluídos os estudos em 15 UF, a saber: Bahia, Santa Catarina, Espírito Santo, Distrito Federal, Goiás, Mato Grosso, Mato Grosso do Sul, Minas Gerais, Paraná, Rio de Janeiro, Rio Grande do Sul, Rondônia, São Paulo, Sergipe e Tocantins, cujos resultados são apresentados nos artigos subsequentes. Além desses 15 artigos, existe um $16^{\circ}$, que explora o impacto da vacinação de bezerras com a B19 na redução da prevalência da brucelose. Excetuando o Estado do Mato Grosso do Sul, todas as demais UF realizaram os trabalhos de campo entre outubro de 2001 e dezembro de 2004. Mato Grosso do Sul optou por aproveitar os resultados do trabalho de campo realizado em 1998.

Apesar do tempo decorrido desde o início das operações de campo, é bastante razoável agrupar os resultados das UF em uma única publicação, dadas as características epidemiológicas da doença, que é de difusão lenta, além da complexidade e dos custos envolvidos nesses estudos, inéditos na história da saúde animal brasileira. Mais ainda, o respeito às limitações das UF para aderir ao projeto foi fundamental para o sucesso das atividades até então desenvolvidas.

\section{REFERÊNCIAS BIBLIOGRÁFICAS}

BAUMGARTEN, D. Brucellosis: A short review of the disease situation in Paraguay. Vet. Microbiol., v.90, p.63-69, 2002.

BRASIL. Ministério da Agricultura e Abastecimento. Diagnóstico de saúde animal, Brasília, 1977. 735p.

BRASIL. Ministério de Agricultura, Pecuária e Abastecimento. Programa Nacional de Controle e Erradicação da Brucelose e da Tuberculose Animal (PNCEBT): Manual técnico. Brasília, 2006. 184p.

BREW, S.D.; PERRETT, L.L.; STACK, J.A. et al. Human exposure to Brucella recovered from a sea mammal. Vet. Rec., v.144, p.483, 1999.

CAPASSO, L. Bacteria in two-millennia-old cheese, and related epizoonoses in Roman populations. J. Infect., v.45, p.122-127, 2002.

CORBEL, M.J. Brucellosis: An overview. Emerg. Infect. Dis., v.3, p.213-221, 1997.

CORBEL, M.J.; ELBERG S.S.; COSIVI, O. (Ed.). Brucellosis in humans and animals. Geneva: WHO Press, 2006. 89p

FOSTER, G.; OSTERMAN B.S.; GODFROID, J. et al. Brucella ceti sp. nov. and Brucella pinnipedialis sp. nov. for Brucella strains with cetaceans and seals as their preferred hosts. Int. J. Syst. Evol. Microbiol., v.57, p.2688-2693, 2007.

GODFROID, J.; CLOECKAERT, A.; LIAUTARD, J.P. et al. From the discovery of the Malta fever's agent to the discovery of a marine mammal reservoir, brucellosis has continuously been a re-emerging zoonosis. Vet. Res., v.36, p.313-326, 2005.

GODFROID, J.; KÄSBOHRER, A. Brucellosis in the European Union and Norway at the turn of the twenty-first century. Vet. Microbiol., v.90, p.135-145, 2002. 
LEÓN, F.C. Brucelosis ovina y caprina. Paris: Office International des Epizooties, 1994. 451p.

LUNA-MARTINEZ, J.E; MEJÍA-TERÁN, C. Brucellosis in Mexico: Current status and trends. Vet. Microbiol., v.90, p.19-30, 2002.

McDONALD, W.L., JAMALUDIN, R., MacKERETH, G. et al. Characterization of a Brucella sp. strain as a marine-mammal type despite isolation from a patient with spinal osteomyelitis in New Zealand. J. Clin. Microbiol., v.44, p.4363-4370, 2006.

McGIVEN, J.; HENDRY, L.; BROWN, D. et al. The improved specificity of bovine brucellosis testing in Great Britain. Res. Vet. Sci., v.84, p.38-40, 2008.

MORENO, E. Brucellosis in Central America. Vet. Microbiol., v.90, p.31-38, 2002.

NICOLETTI, P. A short history of brucellosis. Vet. Microbiol., v.90, p.5-9, 2002.
PAULIN, L.M.; FERREIRA-NETO, J.S. $O$ combate à brucelose bovina: Situação brasileira. Jaboticabal: Funep, 2003. 154p.

POESTER, F.P.; GONÇALVES, V.S.P; LAGE, A.P. Brucellosis in Brazil. Microbiology, v.90, p.55-62, 2002.

RAGAN, V. The Animal and Plant Health Inspection Service (APHIS): Brucellosis eradication program in the Unites States. Vet. Microbiol., v.90, p.11-18, 2002.

SAMARTINO, L.E. Brucelosis in Argentina. Vet. Microbiol., v.90, p.71-80, 2002.

SCHOLZ, H.C.; HUBALEK, Z.; SEDLÁCEK, I. et al. Brucella microti sp. nov., isolated from the common vole Microtus arvalis. Int. J. Syst. Evol. Microbiol., v.58, p.375-382, 2008.

SOHN, A.H.; PROBERT, W.S.; GIASER, C.A et al. Human neurobrucellosis with intracerebral granuloma caused by a marine mammal Brucella spp. Emerg. Infect. Dis., v.9, p.485488, 2003. 